\title{
Apical Hypertrophic Cardiomyopathy in an asymptomatic male
}

\author{
Anish Hirachan ${ }^{1}$, Bishal KC $^{1}$ \\ ${ }^{1}$ Cardiologist, Gautam Buddha Community Heart Hospital, Butwal ,Nepal
}

DOI: https://doi.org/10.3126/jaim.v7i2.23489

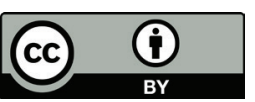

This work is licensed under a Creative Commons Attribution 3.0 Unported License.

\begin{abstract}
Apical Hypertrophic cardiomyopathy is a rare variant of HCM and has a prevalence of around 15\% among HCM patients. As compared to other variants, it has a relatively benign prognosis . Predominantly prevalent in the Japanese population ; it is characterized by localized hypertrophy of the $\mathrm{LV}$ apex as compared to other segments. It is usually silent in early stages and is manifested at an adult stage with typical ECG changes of giant $\mathrm{T}$ wave inversions in the precordial leads. Transthoracic echocardiography remains the mainstay of non - invasive diagnosis . We report a case of an asymptomatic elderly male who presented with the classical deep $\mathrm{T}$ wave inversion in ECG for which echocardiography revealed the apical variant of hypertrophic cardiomyopathy .
\end{abstract}

\section{Introduction}

Apical hypertrophic cardiomyopathy (apical HCM) is an atypical phenotype of non- obstructive HCM (hypertrophic cardiomyopathy) and it is more prevalent in Japanese people. ${ }^{1}$ Of the four common types, apical hypertrophic cardiomyopathy (AHCM) is a rare form, which was first reported in Japan in $1979 .^{2}$ It is often described as a non obstructive form of hypertrophic cardiomyopathy where the hypertrophy predominantly involves the $\mathrm{LV}$ apex .

\section{Case Report}

A 58 year old male presented for cardiovascular evaluation after a suspected ischemic ECG changes from another centre. He denied any history of angina, breathlessness, syncope or palpitations in the past. His pulse rate was $86 / \mathrm{min}$ regular and BP of $130 / 80 \mathrm{mmHg}$ in both arms. No significant findings were noted on general and cardiovascular examination as well. Electrocardiogram revealed deep $\mathrm{T}$ wave inversion in the lateral leads along with features of LVH (fig 1 ). His chest X-ray was normal and other lab parameters were also within normal limits.2D and color echocardiography showed normal left ventricle (LV) size and ejection fraction. He had grade 1 diastolic dysfunction with normal basal septal and posterior wall thickness ( IVS $11 \mathrm{~mm}$, PW $9 \mathrm{~mm}$ ). The salient finding was the presence of severe concentric hypertrophy at the apex, measuring $20 \mathrm{~mm}$ ( both anterior and posterior wall ) with apex to base wall thickness ratio (ABR) of $>1.5$. No intracavitary gradient was recorded on Doppler interrogation. The left atrium was mildly dilated and right heart chambers were within normal limits. The LV cavity showed obliteration in systole without apparent regional wall motion abnormalities.(Figure 2,3)

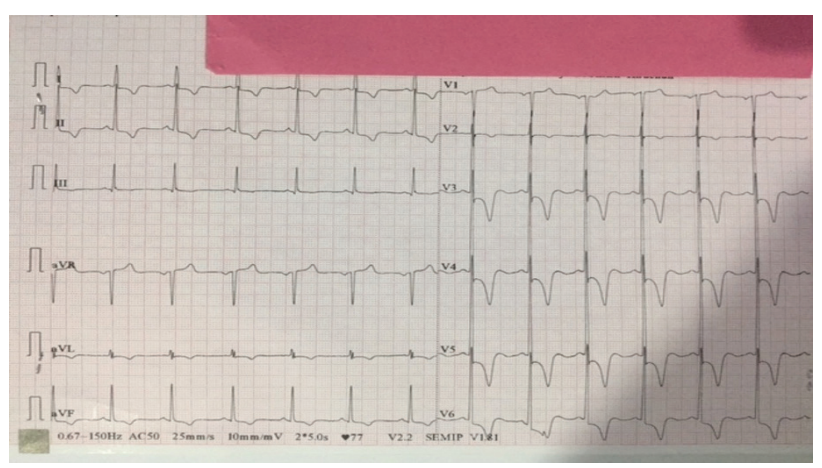

Fig 1: 12 lead ECG showing Giant T - wave inversions in lateral leads

\section{Correspondence Author}

Anish Hirachan

Gautam Buddha Community Heart Hospital,

Butwal, Nepal

Email: hirachananish@gmail.com 


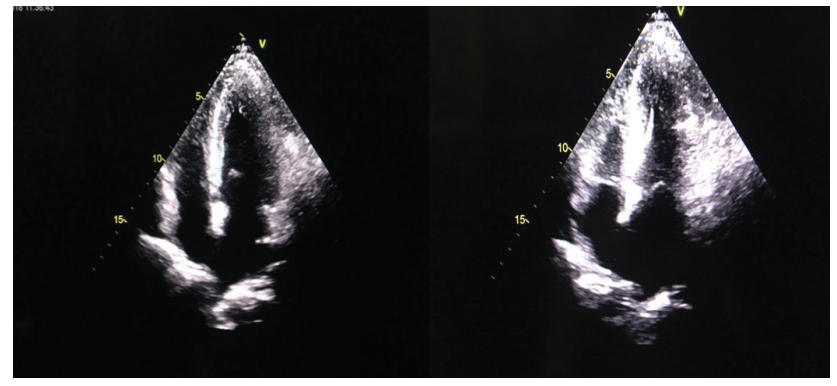

Figure 2 : A4C view showing obliteration of $L V$ apex with hypertrophied muscle

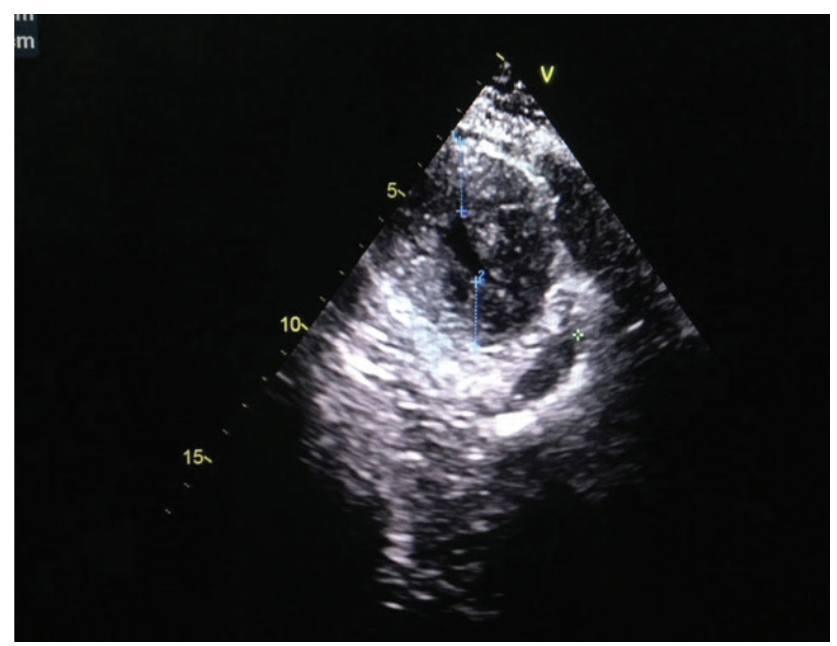

Fig 3 : Short axis echo view showing hypertrophied LV apex ( anterior $20 \mathrm{~mm}$, posterior $20 \mathrm{~mm}$ )

\section{Discussion}

Apical HCM or the Japanese variant (Yamaguchi syndrome) is a rare morphologic variant of HCM in which the hypertrophy of the myocardium predominantly involves the apex of the left ventricle. ${ }^{3}$ This group of patients usually do not have obstructive features ; are clinically silent and also have the most benign prognosis . However, one third of them may present with potential life threatening complications as myocardial infarction , apical infarction with aneurysm, ventricular tachycardia , sudden cardiac death and stroke. ${ }^{4}$

About $54 \%$ of patients with apical HCM are asymptomatic and the most common symptoms are chest pain, followed by palpitation, dyspnea and syncope. In a published large series, these patients presented with atypical chest pain (14\%), palpitation $(10 \%)$, dyspnea $(6 \%)$, presyncope /syncope $(6 \%) .{ }^{5}$ In the absence of epicardial coronary artery disease, patients can have chest pain due to narrowing of intramural coronary vessels due to the hypertrophied myocardium as well as increased myocardial oxygen demand. Ventricular arrhythmias may result from myocardial ischemia and fibrosis as a result of asymmetric hypertrophy . The most frequent ECG findings are classic deep T-wave inversions in the precordial leads found in $93 \%$ of patients and about $65 \%$ of them have LVH. ${ }^{6}$ The most useful noninvasive diagnostic tests for detecting apical HCM is echocardiography and cardiac MRI. The diagnostic criteria for AHCM includes asymmetric LV hypertrophy, confined to the $L V$ apex, with an apical wall thickness $\geq 15 \mathrm{~mm}$ and a ratio of maximal apical to posterior wall thickness $\geq 1.5 \mathrm{~mm} .{ }^{7,8}$ Further, a classical "spade like deformity" can be seen on cardiac ventriculogram due to obliteration of the cardiac apex .

Management of patients with apical variant of HCM includes symptom control with beta blockers, calcium channel blockers for reducing HR and myocardial oxygen demand . In asymptomatic patients, no specific therapy has been outlined, but counseling is certainly recommended for symptomatic monitoring to notice any syncope or presyncopal events on follow up. In other high risk group of patients associated with risk of SCD, ICD implantation should be considered for primary prevention. This case was presented to highlight the importance of focused echocardiography for detecting this rare variant of HCM in patients having the classical ECG changes of apical HCM . This asymptomatic patient was put on beta blockers for his hypertension and asked for a regular follow up along with screening of his family members .

\section{Conclusion}

Diagnosis of apical variant of HCM should always be considered in patients with classical giant $T$ wave inversions on ECG ; even though these patients being mostly asymptomatic . Longstanding prognosis is comparatively better as compared to other phenotypes. However, long term follow up and surveillance data is relatively sparse on this variant of HCM . Hence, these patients need close follow up to prevent sudden cardiac death .

\section{References}

1. Kitaoka H, Doi Y, Casey SA, et al. Compari- son of Prevalence of Apical Hypertrophic Cardiomyopathy in Japan and the United States. Am J Cardiol 2003; 92, 11831186.

2. Sakamoto T, Tei C, Murayama M, et al. Giant T wave inversion as a manifestation of asymmetrical apical hypertrophy (AAH) of the left ventricle. Echocardiographic and ultrasono-cardio tomographic study. Jpn Heart J 1976;17(5):611-629.

3. Arad M, Penas-Lado M, Monserrat L, et al. Gene mutations in apical hypertrophic cardiomyopathy. Circulation. 2005;112(18):2805-2811

4. Kereiakes DJ, Anderson DJ, Crouse L, et al. Apical hypertrophic cardiomyopathy. Am Heart J. 1983;105(5):855-856

5. Eriksson MJ, Sonnenberg B, Woo A, et al. LongTerm Outcome in Patients with Apical Hypertrophic Cardiomyopathy. Jour- nal of American College of Cardiology 2002; 39, 638-645.

6. Choi EY, Rim SJ, Ha JW, et al. Phenotypic spectrum and clinical characteristics of apical hypertrophic cardiomyopathy: multicenter echo-Doppler study. Cardiology 2008;110(1):53-61.

7. Sakamoto T, Amano K, Hada Y, et al. Asymmetric apical hypertrophy: ten years experience. Postgrad Med J. 1986; 62(728):567-570.

8. Stainback RF. Apical hypertrophic cardiomyopathy. Tex Heart Inst J 2012;39(5):747-749. 